\title{
Structure of the human respiratory syncytial virus M2-1 protein in complex with a short positive-sense gene-end RNA
}

\author{
B Liang ${ }^{1}, \mathrm{Y} \mathrm{Gao}^{1}, \mathrm{D} \mathrm{Cao}^{1}$, S Pawnikar ${ }^{2}, \mathrm{Y} \mathrm{Miao}^{2}$ \\ ${ }^{1}$ SOM, Emory University, Atlanta, GA, ${ }^{2}$ University of Kansas, Lawrence, KS \\ bo.liang@emory.edu
}

The M2-1 protein of human respiratory syncytial virus (HRSV) is a zinc-binding transcription anti-terminator that regulates the processivity of the HRSV RNA dependent RNA polymerase (RdRP). Here, we reported a crystal structure of HRSV M2-1 bound to a short positive-sense gene-end RNA (SH7) at $2.7 \AA$ resolution. We identified multiple critical residues of M2-1 involved in RNA interaction and examined their roles using mutagenesis and MicroScale Thermophoresis (MST) assay. We found that hydrophobic residues such as Phe23 are indispensable for M2-1 to recognize the Adenine (A) base of RNA. We also captured spontaneous binding of RNA (SH7) to the M21 protein in all-atom simulations using a robust Gaussian accelerated molecular dynamics (GaMD) method. Both the experiments and simulations revealed that two separate domains of M2-1 interact with RNA, suggesting that the recognition of RNA by the zinc-binding domain (ZBD) and binding of RNA by the core domain (CD) are independent of each other. Collectively, our results provided a structural basis for RNA recognition by HRSV M21.

Acta Cryst. (2020). A76, a117 